\title{
How is China's Health Development During 2012 to 2017? A Longitudinal Study Based on Comprehensive Evaluation and Linear Growth Model
}

\author{
Gangming Zhang \\ Wuhan \\ kelifa Mohammedosman Mohammedhamid \\ Wuhan University \\ Fang Tang \\ Wuhan University \\ Jiaye Xu \\ Wuhan University \\ Bhawana Shrestha \\ Wuhan University \\ Peigang Wang ( $\sim$ wpg926@whu.edu.cn ) \\ Wuhan University, China https://orcid.org/0000-0003-4398-0689
}

Research article

Keywords: Health development; Comprehensive evaluation; Multilevel model; China; Influencing factors for health development

Posted Date: May 28th, 2020

DOI: https://doi.org/10.21203/rs.3.rs-30172/v1

License: (c) (i) This work is licensed under a Creative Commons Attribution 4.0 International License. Read Full License 


\section{Abstract}

Background: Since 2012, China has come into a new period of health development. This paper comprehensively described China's health development and explored associated influencing factors during the last 6 years (2012-2017).

Methods: Data for this study came from statistics yearbook and analysis unit were provincial regions. Comprehensive evaluation (principal component analysis and entropy weight method) was employed to calculate the comprehensive health index to evaluate the health. Then linear growth model was applied to explore factors that influenced the development.

Results: Results showed that, since 2012, China's health had a sustainable growth but inequities among provincial regions were still existing and becoming larger. For influencing factors, time was always a significant positive predictor ( ) and it was affected by geographical distributions ( ) and distance to Beijing ( ), which indicated that regions in central or far from Beijing had a lower growth. Among socioeconomics variables, urbanization level was the final factor ( ) that promoted China's health development and caused development inequalities. Besides, health level was also influenced by the distance to Beijing ( ), regions near to Beijing had a higher health level.

Conclusion: Time effect was the results of policy, during 2012 to 2017, China's sustainable health development was brought by the policy effect and growth of urbanization level. Thus, it is crucial for one country to introduce suitable health policies and narrow the urban-rural gaps to improve its health. Distance to Beijing represented the potential political influence on health of one country's capital due to the policy execution level which means that supervisions need to be strengthened for regions far from the capital. Besides, supports and supervisions also need to be enhanced in central regions. They were one of the reasons that caused development inequalities.

\section{Full Text}

This preprint is available for download as a PDF.

\section{Tables}

Table 1 Index system of provincial-level health 


\begin{tabular}{|c|c|c|}
\hline Indicators & Unit & Type \\
\hline \multicolumn{3}{|l|}{ Health condition } \\
\hline Maternal Mortality Rate & $1 / 100000$ & Negative \\
\hline Infant Mortality Rate & $\%$ & Negative \\
\hline Perinatal mortality Rate & $\%$ & Negative \\
\hline Expectation of life & year & Positive \\
\hline Infectious disease morbidity & $1 / 100000$ & Negative \\
\hline \multicolumn{3}{|l|}{ Health workforce } \\
\hline Density of general practitioner & $1 / 10000$ & Positive \\
\hline Density of certified doctor & $1 / 1000$ & Positive \\
\hline Density of certified nurse & $1 / 1000$ & Positive \\
\hline Density of medical technological personnel & $1 / 1000$ & Positive \\
\hline Density of traditional Chinese medicine personnel & $1 / 1000$ & Positive \\
\hline \multicolumn{3}{|l|}{ Health service availability } \\
\hline Average number of hospital visits & Person-time & Positive \\
\hline Average number of traditional Chinese medicine hospital visits & Person-time & Positive \\
\hline \multicolumn{3}{|l|}{ Health facility } \\
\hline Density of general hospital & $1 / 100000$ & Positive \\
\hline Density of primary healthcare institution & $1 / 10000$ & Positive \\
\hline Density of public health institution & $1 / 100000$ & Positive \\
\hline Density of traditional Chinese medicine hospital & & Positive \\
\hline General hospital bed density & $1 / 1000$ & Positive \\
\hline Primary healthcare institution bed density & $1 / 1000$ & Positive \\
\hline Public health institution bed density & $1 / 1000$ & Positive \\
\hline Total expenditure on health as a percentage of gross domestic product & $\%$ & Positive \\
\hline Per capita total expenditure on health & $\mathrm{CNY}$ & Positive \\
\hline \multicolumn{3}{|l|}{ Environment health } \\
\hline Average area of urban green space & $\mathrm{m}^{2}$ & Positive \\
\hline Emission intensity of industrial waste & - & Negative \\
\hline Emission intensity of exhaust gas & - & Negative \\
\hline Emission intensity of ammonia nitrogen in exhaust water & - & Negative \\
\hline
\end{tabular}

Table 2. Average value of provincial-level CHI from 2012 to 2017

\begin{tabular}{ccccccc}
\hline Years & 2012 & 2013 & 2014 & 2015 & 2016 & 2017 \\
\hline CHI & 48.2157 & 51.6772 & 52.7700 & 54.1344 & 56.0014 & 58.0418 \\
PCA_CHI & 47.7543 & 48.8561 & 48.9800 & 49.4270 & 51.0686 & 52.4669 \\
EWM_CHI & 48.6770 & 54.4983 & 56.5601 & 58.8419 & 60.9342 & 63.6166 \\
CHI SD & 10.3755 & 12.7654 & 10.0528 & 10.4866 & 10.8912 & 11.0452 \\
\hline Years & $2012-2013$ & $2013-2014$ & $2014-2015$ & $2015-2016$ & $2016-2017$ \\
\hline CHI Growth Rate & $7.18 \%$ & $2.11 \%$ & $2.59 \%$ & $3.45 \%$ & $3.64 \%$ \\
\hline
\end{tabular}


Note: CHI: Comprehensive health index, PCA_CHI: Comprehensive health index calculated by principal component analysis, EWM_CHI: Comprehensive health index calculated by entropy weight method, and CHI SD: Standard deviations of comprehensive health index.

Table 3 CHI of Eastern, Central and Western Regions During 2012 to 2017

\begin{tabular}{cccccccc}
\hline Years & 2012 & 2013 & 2014 & 2015 & 2016 & 2017 & Average \\
\hline Eastern Region & 56.53 & 62.27 & 60.81 & 63.19 & 65.34 & 67.66 & 62.63 \\
Central Region & 44.95 & 46.30 & 48.54 & 49.48 & 50.90 & 52.34 & 48.75 \\
Western Region & 42.77 & 45.55 & 48.23 & 48.94 & 50.85 & 53.02 & 48.23 \\
\hline Whole Country & 48.22 & 51.68 & 52.77 & 54.13 & 56.00 & 58.04 & 53.47 \\
\hline
\end{tabular}

Note: Eastern region includes Beijing, Tianjin, Hebei, Shanghai, Shandong, Jiangsu, Zhejiang, Guangdong, Liaoning, Fujian and Hainan; Central region includes Heilongjiang, Jilin, Henan, Shanxi, Anhui, Hunan, Hubei and Jiangxi; Western region includes Inner Mongolia, Shaanxi, Xinjiang, Tibet, Qinghai, Gansu, Ningxia, Guizhou, Yunnan, Sichuan, Chongqing and Guangxi.

Table 4 Factors influencing provincial-level health development 


\begin{tabular}{|c|c|c|c|c|}
\hline Variables & Model 1 & Model 2 & Model 3 & Model 4 \\
\hline Intercept & $58.0067(1.9805) * * *$ & $67.4357(2.6297)^{* * *}$ & $44.7950(5.3109) * * *$ & $26.0916(7.1580)^{* * *}$ \\
\hline Time & $1.8134(0.113)^{* * *}$ & $1.9215(0.186)^{* * *}$ & $2.4012(0.4781)^{* * *}$ & $2.222(0.4336) * * *$ \\
\hline Western & & $-14.3612(3.6407) * * *$ & $-3.0437(3.5304)$ & $-0.7746(3.4673)$ \\
\hline Central & & $-14.9954(4.0527) * * *$ & $-7.481(3.5403)$ & $-5.4883(3.4826)$ \\
\hline Time*Western & & $0.0177(0.2575)^{*}$ & $-0.0706(0.2992)$ & $-0.4261(0.2775)$ \\
\hline Time*Central & & $-0.4452(0.2866) *$ & $-0.2570(0.2938)$ & $-0.5628(0.2697)^{*}$ \\
\hline PerGDP & & & $0.9666(0.4473)^{*}$ & $0.4084(0.4182)$ \\
\hline Distance & & & $3.0149(1.3759)^{*}$ & $1.7918(1.3837)^{*}$ \\
\hline Time*Distance & & & $-0.3081(0.1197)^{*}$ & $-0.2661(0.1066)^{*}$ \\
\hline PopuDensity & & & $0.005(0.002)^{*}$ & $0.0023(0.002)$ \\
\hline UrbanPopu & & & & $0.4284(0.11)^{* * *}$ \\
\hline \multicolumn{5}{|c|}{ Random effects parameters } \\
\hline Variance (intercept) & $120.11(31.3955)^{* * *}$ & $74.5925(20.3318) * * *$ & $41.4175(12.3497) * * *$ & $39.51(11.4312)^{* * *}$ \\
\hline Variance (time) & $0.2347(0.1043)^{*}$ & $0.2192(0.1037)^{*}$ & $0.1945(0.1034) *$ & $0.1152(0.0787)$ \\
\hline Covariance & $0.1009(1.2714)$ & $-0.5337(1.0184)$ & $-0.8458(0.8449)$ & $1.4118(0.7661)$ \\
\hline \multicolumn{5}{|l|}{ (intercept, time) } \\
\hline Variance (residual) & $2.8232(0.3585)^{* * *}$ & $2.8232(0.3585)^{* * *}$ & $2.7385(0.3487)^{* * *}$ & $2.7957(0.3573)^{* * *}$ \\
\hline$-2 \mathrm{LL}$ & 918.1 & 891.9 & 873.5 & 864.0 \\
\hline AIC & 926.1 & 899.9 & 881.5 & 872.0 \\
\hline BIC & 931.8 & 905.7 & 887.3 & 877.7 \\
\hline
\end{tabular}

Note: $* \mathrm{P}<0.05, * * \mathrm{P}<0.01, * * * \mathrm{P}<0.001$

\section{Figures}




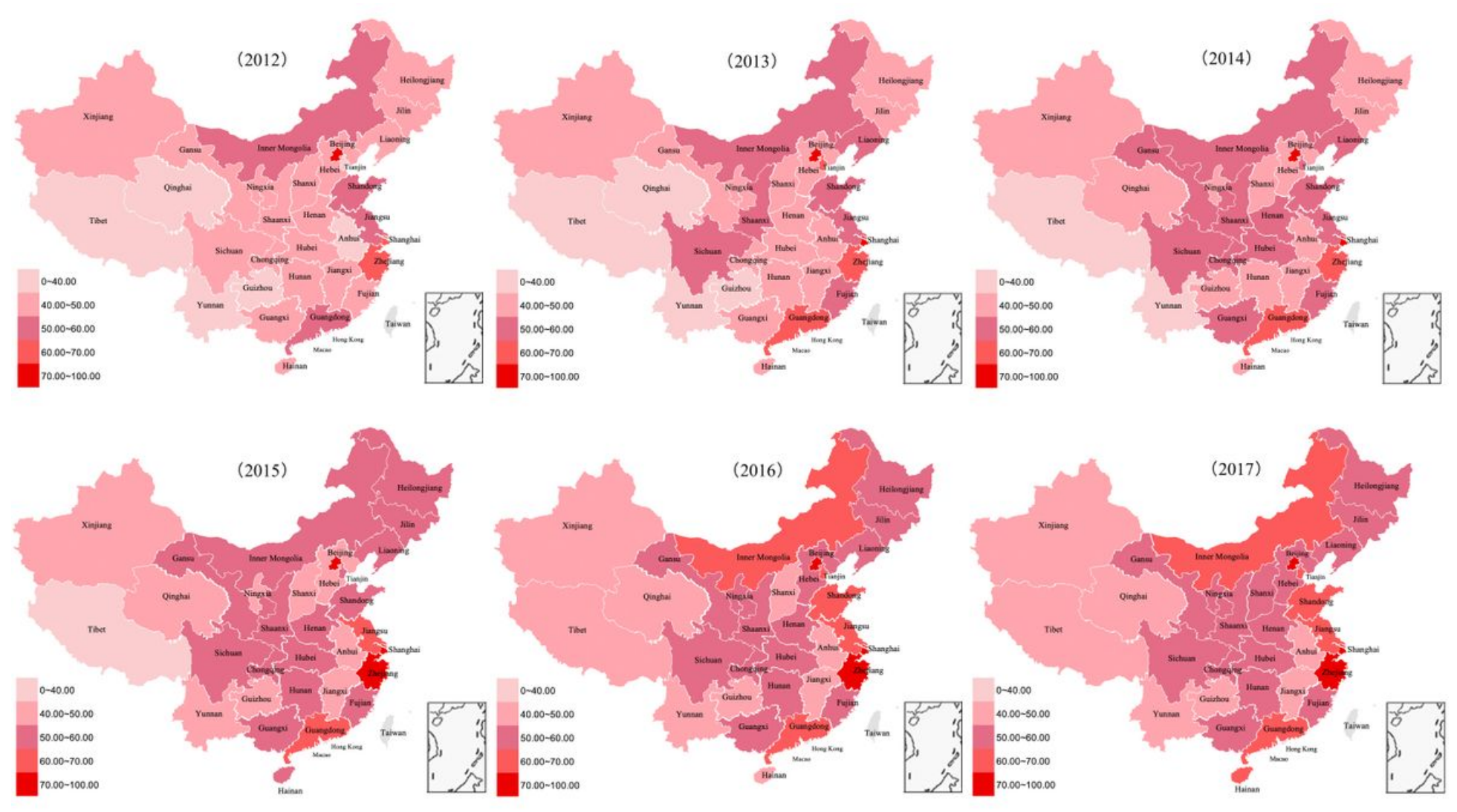

\section{Figure 1}

Space distribution of China's CHI from 2012 to 2017. This map showed the space distribution of China's $\mathrm{CHI}$ during 2012 to 2017. As we can find in this map, deeper of the color (red) represented a higher $\mathrm{CHI}$ score. From 2012 to 2017, scores of most provincial regions showed a growth trend and were also higher in eastern regions. Provincial regions near Beijing, China's capital, also were higher than those far from it. Central regions, like Anhui and Jiangxi, had evident lower growth rate than others.Data was only limited for mainland China, there was no data for Taiwan, Hong Kong and Macao (the color were grey). Note: The designations employed and the presentation of the material on this map do not imply the expression of any opinion whatsoever on the part of Research Square concerning the legal status of any country, territory, city or area or of its authorities, or concerning the delimitation of its frontiers or boundaries. This map has been provided by the authors. 